\title{
DESENVOLVIMENTO DE UM SIMULADOR DE TEMPERATURAS DOS REVESTIMENTOS REFRATÁRIOS DE UM FORNO DE AQUECIMENTO *
}

\section{Resumo}

Rafael Duclou Rito ${ }^{1}$ Lis Nunes Soares ${ }^{2}$

Ricardo Junqueira Silva ${ }^{3}$

Com temperaturas altíssimas atingidas, os fornos são equipamentos responsáveis pelo aquecimento de materiais na produção de tubos sem costura. Assim, o investimento requerido de um forno em bom funcionamento é quase todo em combustível. Este trabalho foi desenvolvido objetivando a qualidade dos produtos e a otimização dos períodos em que o forno não produz. Durante os períodos citados, o forno é desligado ou mantido a uma temperatura menor que a de operação. Estudando a transferência de calor, principalmente no refratário, é possível analisar a melhor prática em situações improdutivas. Portanto, foi desenvolvido um simulador da temperatura dos refratários ao longo do tempo do forno em questão, sendo possível espelhar parâmetros reais para encontrar o controle de temperatura ótimo do forno, em que a temperatura da atmosfera interna é a principal entrada no programa. Termopares foram instalados ao longo das paredes refratárias para que o modelo fosse validado e aprimorado. Paralelamente, pontuou-se a relação dos materiais enfornados com as medições dos termopares, a fim de encontrar uma temperatura ideal de enfornamento. Os resultados foram positivos e permitem o uso de artifícios de refinamento dos resultados.

Palavras-chave: Forno Rotativo; Revestimento refratário; Transferência de calor; Simulador de temperaturas.

\section{A TEMPERATURE SIMULATOR OF THE REFRACTORY LINING FROM A HEATING FURNACE}

\section{Abstract}

Furnaces are equipments responsible for heating materials in the production of seamless pipes and tubes. With very high temperatures achieved, the money input for a well functioning furnace is almost all spent on fuel. This work was developed aiming the product quality and the optimization of periods when the furnace isn't producing. During these periods, the furnace is turned off or sustained at a temperature lower than the operating one. Studying the heat transfer, above all in the refractory, it is possible to analyze the best practice for unproductive periods. Therefore, a temperature simulator over time of the refractory lining was developed for the furnace in question, being possible to represent real parameters to find the optimal furnace temperature control, in which the internal atmosphere is the main input. Thermocouples were installed to validate and improve the model. At the same time, the relation between the product inside the furnace and the thermocouples results was studied, objectifying an ideal temperature to start processing the materials inside the furnace. The results were positive and also open the possibility to mathematical artifices which can highly improve the accuracy of the results.

Keywords: Refractory lining; Heat transfer; Temperature Simulator; Circular furnace.

1 Estudante de engenharia mecânica, Estagiário, Superintendência de Tecnologia e Engenharia de Processos, Vallourec Soluções Tubulares do Brasil S.A., Belo Horizonte, Minas Gerais, Brasil.

2 Engenheira Mecânica, Doutora, Engenheira de Processos Sênior, Superintendência de Tecnologia e Engenharia de Processos, Vallourec Soluções Tubulares do Brasil S.A., Belo Horizonte, Minas Gerais, Brasil.

3 Engenheiro Mecânico, Doutor, Engenheiro de Processos Sênior, Superintendência de Tecnologia e Engenharia de Processos, Vallourec Soluções Tubulares do Brasil S.A., Belo Horizonte, Minas Gerais, Brasil. 


\section{INTRODUÇÃO}

$\mathrm{Na}$ produção de tubos laminados sem costura, é preciso aquecê-los a elevadas temperaturas antes do processo de laminação. Tais temperaturas são alcançadas graças ao forno, robusto equipamento térmico que aproveita o alto poder calorífico de determinados combustíveis para aquecer seu interior, devidamente isolado por paredes refratárias.

Visando solucionar os problemas de qualidade nos tubos e aperfeiçoar os períodos sem produção nos fornos, que se tornaram mais recorrentes, graças à recessão econômica dos últimos anos, estudou-se o comportamento térmico dos refratários dos mesmos.

O resultado foi obtido na modelagem do forno: um simulador das temperaturas dos refratários, do consumo de gás combustível e dos tempos de aquecimento e resfriamento.

Um primeiro trabalho foi realizado, a princípio em um forno retangular de tratamento térmico. Partindo deste, o modelo foi ampliado para um operar um forno circular de aquecimento. Extrapolando a motivação inicial, outras demandas surgiram para 0 simulador, aproveitando principalmente os resultados da simulação das temperaturas de refratário do forno.

O desenvolvimento da modelagem foi concluído com o estudo das relações de transferência de calor no interior e exterior do forno e a condução nas paredes refratárias.

\section{MATERIAIS E MÉTODOS}

O processo de transferência de calor envolvendo o forno de aquecimento em questão. $O$ ambiente externo também foi profundamente estudado para 0 desenvolvimento deste estudo.

\section{RESULTADOS E DISCUSSÃO}

\subsection{O Modelo}

O forno de aquecimento considerado é circular, com revestimento interno composto por cimento refratário e fibra de cerâmica. Externamente, há a estrutura metálica.

A modelagem do forno levou em conta as relações de transferência de calor com o forno improdutivo, vazio. O objetivo é obter o comportamento da temperatura ao longo das camadas refratárias.

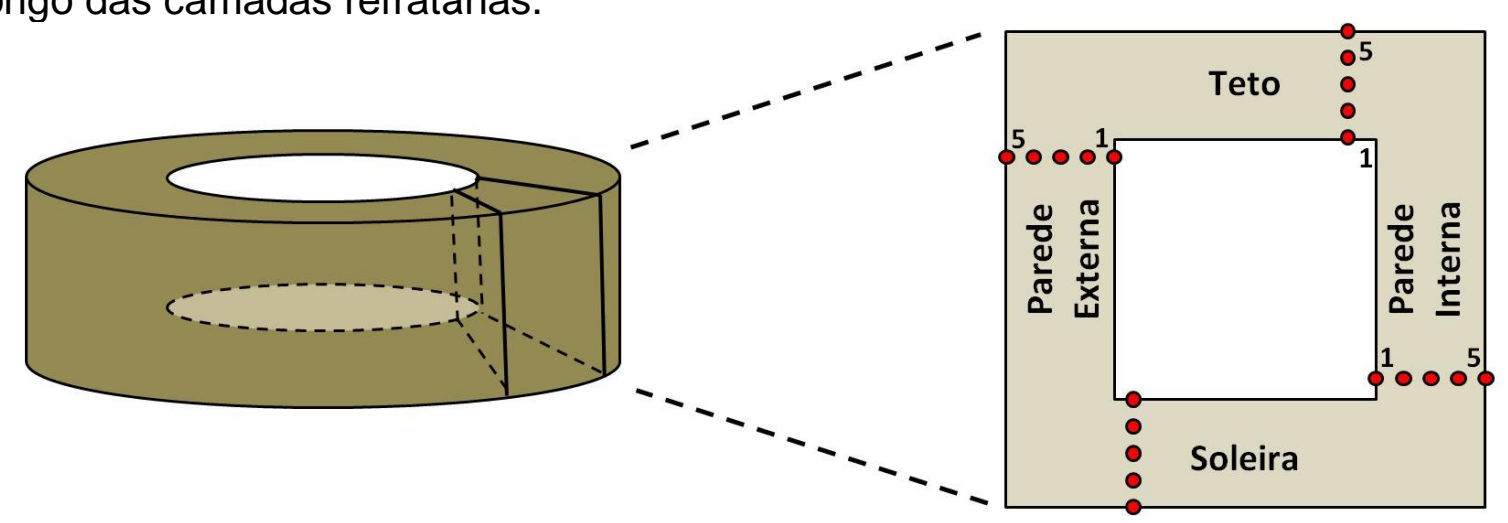

Figura 1. O modelo do forno circular considerado. 
Cada parte do forno, - teto, soleira e paredes - é dividida por cinco pontos igualmente espaçados. O modelo calcula uma temperatura para cada ponto, através de balanços energéticos ao longo do tempo. A Figura 1 indica o sentido usado na nomenclatura de cada temperatura. Estas são nomeadas pela letra "T" e dois números. Como mostrado a seguir, o primeiro índice numérico define a divisão do forno, e o segundo é contado a partir da atmosfera interna, indicando cada camada refratária, em ordem crescente, de um a cinco. Assim, as extremidades de número um e cinco situam-se em contato com as atmosferas internas e externas, respectivamente.

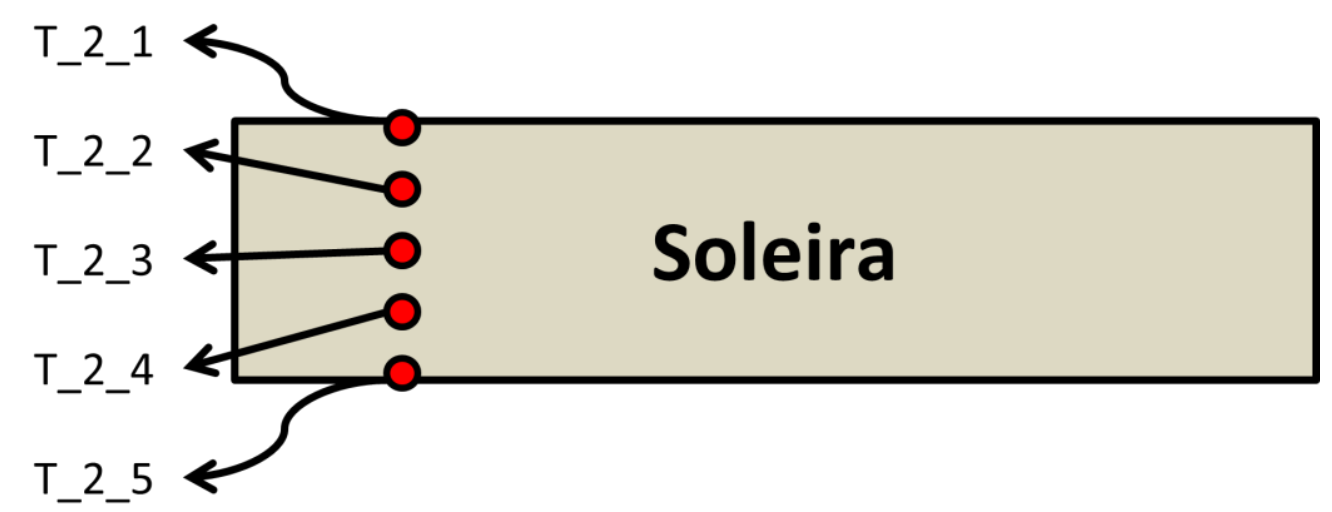

Figura 2. Exemplo de cada temperatura na soleira.

Tabela 1. Índices usados para cada divisão do forno

\begin{tabular}{cc}
\hline$T_{1}$ & Teto \\
\hline$T_{2} 2$ & Soleira \\
\hline$T_{3} 3$ & Parede Externa \\
\hline$T_{4} 4$ & Parede Interna \\
\hline
\end{tabular}

A fim de não congestionar o programa e simplificar os cálculos, algumas considerações foram levadas em conta pelo simulador:

- Conforme a Figura 1, as geometrias internas e externas do forno são simplificadas.

- As temperaturas da chama de combustão e do gás no interior do forno são iguais.

- A partir da inércia térmica do refratário obtiveram-se valores discretos do tempo e dos materiais das quatro divisões do forno.

- Não foi considerada variação da capacidade térmica dos materiais em questão, em relação à temperatura.

\subsection{Formulação Matemática}

A Figura 3 indica todos os fluxos de calor nas extremidades, por convecção e radiação. A massa de gás e a chama de combustão têm seus fenômenos descritos na convecção e radiação interna dos gases. Internamente, ainda há a indicação da radiação das demais divisões, que quantifica o fluxo de calor em uma divisão, advindo das outras três. Na soleira, por exemplo, leva-se em conta a influência radiação do teto e a das duas paredes. 
No ambiente externo, os gases da atmosfera ambiente estão contabilizados pelos fluxos de convecção e radiação externa dos gases.

Internamente, considerou-se a condução de calor nas paredes dos refratários de todas as divisões: soleira, teto, parede externa e interna.

\section{Atmosfera do}

Forno

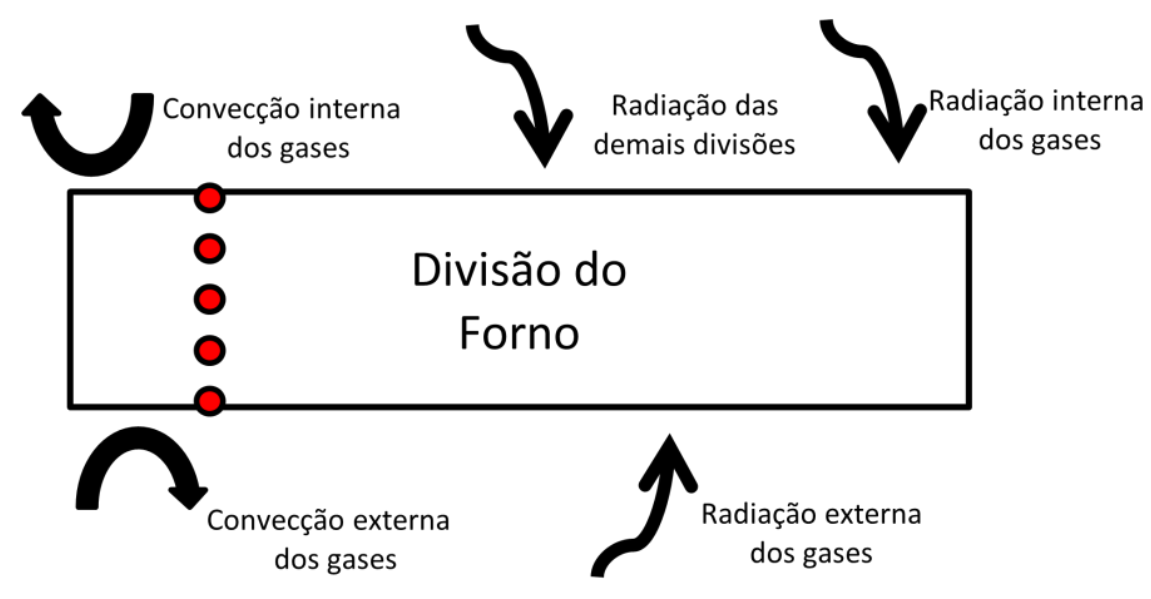

\section{Atmosfera}

\section{Ambiente}

Figura 3. Indicação dos fluxos de calor excetuando condução.

$$
\begin{aligned}
& \varphi_{\text {gas } \rightarrow 1}=\left(h_{g a s}^{r}+h_{\text {int }, 1}^{C C}\right) *\left(T_{\text {gas }}-T_{1 \_1}\right) \\
& \varphi_{\text {gas } \rightarrow 2}=\left(h_{\text {gas }}^{r}+h_{\text {int }, 2}^{C C}\right) *\left(T_{\text {gas }}-T_{2 \_1}\right) \\
& \varphi_{\text {gas } \rightarrow 3}=\left(h_{\text {gas }}^{r}+h_{\text {int }, 3}^{C C}\right) *\left(T_{\text {gas }}-T_{3 \_1}\right) \\
& \varphi_{\text {gas } \rightarrow 4}=\left(h_{\text {gas }}^{r}+h_{\text {int }, 4}^{C C}\right) *\left(T_{\text {gas }}-T_{4_{-} 1}\right) \\
& \varphi_{2,3,4 \rightarrow 1}=h_{1}^{r} *\left(T_{1}-T_{f 1}\right) \\
& \varphi_{1,3,4 \rightarrow 2}=h_{2}^{r} *\left(T_{2}-T_{f 2}\right) \\
& \varphi_{1,2,4 \rightarrow 3}=h_{3}^{r} *\left(T_{3}-T_{f 3}\right) \\
& \varphi_{1,2,3 \rightarrow 4}=h_{4}^{r} *\left(T_{4}-T_{f 4}\right) \\
& \varphi_{1 \rightarrow \infty}=h_{\text {ext }, 1}^{r} *\left(T_{1 \_5}-T_{\infty}\right) \\
& \varphi_{2 \rightarrow \infty}=h_{\text {ext }, 2}^{r} *\left(T_{2,5}-T_{\infty}\right) \\
& \varphi_{3 \rightarrow \infty}=h_{\text {ext } 3}^{r} *\left(T_{35}-T_{\infty}\right) \\
& \varphi_{4 \rightarrow \infty}=h_{\text {ext, } 4}^{r} *\left(T_{4 \_5}-T_{\infty}\right) \\
& \varphi_{1}=-\lambda_{1 \mathrm{med}} \frac{\partial T_{1}}{\partial x} \\
& \varphi_{2}=-\lambda_{2 \text { med }} \frac{\partial T_{2}}{\partial x} \\
& \varphi_{3}=-\lambda_{3 \operatorname{med}} \frac{\partial T_{3}}{\partial x} \\
& \varphi_{4}=-\lambda_{4 \operatorname{med}} \frac{\partial T_{4}}{\partial x}
\end{aligned}
$$


Para cada equação, $\varphi_{\text {gas } \rightarrow x}$ é o fluxo de calor do gás para a divisão indicada por $x$; $h_{g a s}^{r}$ indica o coeficiente de radiação do gás; $h_{i n t, x}^{C C}$ a convecção do ambiente do forno para a superfície indicada; $T_{g a s}$ é a temperatura do gás.

O fluxo de calor de três divisões para uma quarta é $\varphi_{y, w, z} \rightarrow x ; h_{x}^{r}$ é o coeficiente de radiação, recebido pela divisão indicada por $x$, e emanada pelas demais; $T_{f x}$ é a temperatura média da superfície das três divisões, com exceção de $x$.

O fluxo de calor da divisão indicada para o ambiente é $\varphi_{x \rightarrow \infty} ; h_{\text {ext,x }}^{r}$ é o coeficiente de radiação externo do forno e $T_{\infty}$ a temperatura deste mesmo ambiente.

A condução é representada por $\varphi_{x}$, fluxo de calor; $\lambda_{\mathrm{x} \text { med }}$ a condutividade térmica média da divisão indicada e $\frac{\partial T_{x}}{\partial x}$ a variação de temperatura em relação à espessura da divisão considerada.

Com a finalidade de determinar o perfil das temperaturas do gás e do refratário do teto, paredes e soleiras, é necessário resolver as equações, indicadas respectivamente: um balanço energético transiente sobre o volume de gás no forno, a conservação do fluxo de calor através de cada camada de cada divisão do forno e um balanço transiente no interior do material refratário de cada divisão do forno, soleira, teto e paredes, com os fluxos de condução.

Atmosfera do forno:

Superfícies internas $-T_{-} x_{-} 1$ :

$$
m_{g a s} C_{p, g a s} \frac{\partial T_{g a s}}{\partial t}=\varphi_{1 \rightarrow g a s}+\varphi_{2 \rightarrow \text { gas }}+\varphi_{3 \rightarrow \text { gas }}+\varphi_{4 \rightarrow \text { gas }}
$$

Superfícies externas $-T_{-} x_{-} 5$ :

$$
\varphi_{g a s \rightarrow x}+\varphi_{y \rightarrow x}+\varphi_{w \rightarrow x}+\varphi_{z \rightarrow x}=\varphi_{x}
$$

Condução interna:

$$
\varphi_{\infty \rightarrow x}=\varphi_{x}
$$

$$
\frac{\partial T_{x}}{\partial t}=\frac{\lambda_{\mathrm{x}}}{\rho_{1} C_{p, x}} \frac{\partial^{2} T_{x}}{\partial x^{2}}
$$

$\rho_{1}$ é a massa específica e $C_{p, x}$ a capacidade térmica dos revestimentos refratários. $\frac{\partial T_{x}}{\partial t}$ é a variação de temperatura em relação ao tempo.

Entende-se ainda que $x$ é a divisão do forno em interesse, em que se calcula o fluxo de calor, por exemplo.

\subsection{Aquisição de Dados}

A fim de aprimorar e validar os resultados das equações, todas as informações foram coletadas de maneira experimental e com o forno improdutivo.

Termopares foram instalados, em determinadas profundidades, em cada divisão do forno, ou seja, na soleira, no teto e nas paredes internas e externas. Assim, foi possível analisar o perfil de temperaturas ao longo do tempo.

Os dados foram coletados em intervalos de dez minutos e armazenados em loggers. As profundidades foram determinadas a partir das camadas refratárias do forno e do processo dos materiais enfornados.

O forno de aquecimento é dividido em oito regiões. Cada região pode apresentar diferença de temperaturas. Há ainda a soleira do forno, móvel, que rotaciona em torno do próprio eixo. Dois conjuntos de termopares estão posicionados, diametralmente opostos, com cinco termopares em cada conjunto, na divisão do forno soleira. Os termopares das demais divisões estão posicionados em uma determinada zona. 
Os resultados obtidos dos termopares permitiram uma análise profunda do comportamento dos refratários. As espessuras são medidas a partir do exterior do forno.

Durante a campanha de medições, estudou-se paralelamente se alguma das divisões teria maior criticidade que as outras. Com as medições dos termopares instalados, nota-se a inércia térmica de cada divisão. Por ser a mais encorpada, a soleira mostrou-se a divisão com aquecimento mais lento em relação às outras. Ou seja, quaisquer temperaturas esperadas em quaisquer pontos dos refratários serão sempre atingidas por último na segunda - a soleira. Além do mais, a velocidade de rotação da divisão de número dois mostrou um impacto desconsiderável na variação da temperatura de tal refratário.

Assim, o foco inicial das análises de resultado manteve-se em tal divisão, uma vez que se esta é bem descrita é possível controlar todo o processo e atingir o objetivo do estudo. Ou seja, garantidos os resultados da soleira, garantia-se o restante do forno.

\subsection{Validação do Modelo}

Partindo da média das temperaturas das zonas intermediárias do forno, que de fato não diferem muito entre si e são as mais influentes para o gás interno, obteve-se o perfil de todas as divisões. Assim, é possível comparar os cenários reais com os modelados.

Apesar dos termopares estarem em diferentes espessuras do que as que o modelo considera nos cálculos, algumas são muito próximas. E para as que não são, uma ferramenta foi utilizada para projetar as temperaturas de simulação às mesmas profundidades que os termopares.

A comparação de resultados entre o modelo matemático e os valores de temperatura medidos foi feita considerando o perfil de temperatura da atmosfera interna durante o período de uma semana. Uma média foi feita entre as temperaturas de todas as zonas, com exceção das zonas 1 e 8 . Tais zonas apresentam valores próximos uns dos outros e maior impacto no aquecimento do forno. O erro relativo variou para cada termopar, mas, em média, obteve-se erro relativo de $25 \%$, sendo $35 \%$ o maior erro e para uma das temperaturas da soleira, o erro foi menor que $3 \%$.

\subsection{Desenvolvimento Computacional - Simulação de Temperaturas}

Uma ferramenta de simulação computacional foi desenvolvida, aplicando o modelo matemático que resulta no perfil de temperatura dos refratários do forno.

O modelo parte da atmosfera interna do forno. A partir dessas entradas, o programa resolve as equações e retorna as temperaturas de cada divisão e espessura do forno. Então, é possível gerar e comparar quaisquer cenários de perfis de temperatura dos refratários, quando a temperatura interna é mantida, aumentada ou reduzida.

Se há maior rigor em espelhar a temperatura da atmosfera - entrada no modelo, os resultados apresentam menor erro. Com o intuito de obter uma visão geral, sabendo que na prática, muitas vezes, não é possível tamanha dedicação na análise da temperatura da atmosfera, faz-se uma simulação com uma temperatura intermediária, e sendo assim, o erro relativo é maior. 
Sobre economia de combustível, principalmente, avaliou-se a relação entre as temperaturas dos termopares e a qualidade dos produtos para determinar pontos ótimos para o processo.

Para a situação de ligamento ou aquecimento do forno, em que a temperatura da atmosfera interna é incrementada, a melhor prática é que as temperaturas dos termopares atinjam valores constantes, garantido que a energia do forno seja direcionada exclusivamente para o produto.

Estudou-se que quando o último ponto da soleira ( $T$ _2_5) atinge determinada temperatura, o forno está adequado para o enfornamento, garantido a qualidade dos produtos. Tal temperatura corresponde a certa porcentagem da temperatura em condição de regime permanente.

Quando o forno encontra-se vazio, a ferramenta de simulação permite acompanhar o perfil de temperaturas dos refratários. Assim, permite planejar as melhores práticas para a linha de produção, de maneira que o consumo do forno seja o mínimo possível para que o produto seja enfornado e mantenha as propriedades esperadas ao fim do processo.

\section{CONCLUSÃO}

Os valores de calculados apresentaram erro relativo considerável em relação às temperaturas coletadas, se analisamos o processo como um todo. Minuciosamente, os erros podem ser causas de incongruências de entrada do modelo com dados reais. Há faixas de temperaturas, todavia, que o simulador foi totalmente fiel aos resultados dos termopares.

Sabendo disto, o simulador já apresenta potencial de ganho. Porém, pelas considerações da parede nos cálculos, a complexidade do projeto maior, e ainda é possível um refinamento dos resultados, usando outras ferramentas matemáticas.

O estudo dos materiais que são enfornados, e a relação deles com as temperaturas dos termopares ao longo do refratário, também apresentam alto potencial de ganho, visto que sempre há alguma temperatura ótima no refratário para o início do enfornamento. É evidente, então, que conhecendo tais pontos ótimos não há necessidade de maior aquecimento do forno, motivo que causaria desperdício de tempo e combustível.

A ferramenta de simulação foi bem sucedida para trazer, no mínimo, a ordem de grandeza das temperaturas do forno. Algumas temperaturas ainda podem ser referência na análise do processo por apresentaram baixo erro relativo.

Atualmente, há uma versão em desenvolvimento que se mostrou capaz de refinar todos os resultados, com o auxílio de uma ferramenta matemática.

\section{Agradecimentos}

À empresa Vallourec Soluções Tubulares do Brasil S.A. (VSB) por apoiar projetos de pesquisa e desenvolvimento.

À Dra. Lis Nunes Soares e ao Dr. Ricardo Silva Junqueira, engenheiros especialistas em processos térmicos, pela orientação durante o trabalho.

À toda equipe dos setores de Tratamento Térmico, Combustão e Manutenção de Refratários da Laminação Contínua da VSB, na Usina Barreiro, pelo apoio.

Aos colegas de trabalho Fernanda da Silva Machado, Riane Angeli Lopes e Luis Fernando Oliveira Rodrigues, pelo suporte em várias etapas do projeto. 


\section{REFERÊNCIAS}

1 Trinks, W..Mawhinney ,M.H..Shannon, R.A..Reed, R.J..Garvey, J.R.. Industrial Furnaces, sixth edition, Wiley - John Wiley and sons, INC., Hoboken, New Jersey 2004..

2 Taine J., lacona E., Petit J-P..Transferts Thermiques - Introduction aux transferts d'énergie, quatrième édition, $4^{\text {a }}$ édition, Dunod., Paris 2008.

3 E.P. Keramidaa, H.H. Liakosa, M.A. Fountib, A.G. Boudouvisa,N.C. Markatosa. "Radiative heat transfer in natural gas-fired furnaces", International Journal of Heat and Mass Transfer 43. 2000.

4 Incropera, F.P.. Dewitt, D.P. .Fundamentos de Transferência de Calor e de Massa, 3a edição, LTC - Livros Técnicos e Científicos Editora S. A., R. J. 1990.

5 Mochida A, Kudo K, Mizutani Y, Hattori M, Nakamura Y. "Transient Heat Transfer Analysis in Vacuum Furnaces Heated by Radiant Tube Burners". Energy Conversion and Management Vol. 38, No. 10-13, pp 1169-1176. 1997.

6 De Loze Arnaud-Guilhem, Rodrigues Luís Fernando, Soares Lis. "Desenvolvimento de modelo matemático para transferência de calor no revestimento refratário de um forno de tratamento térmico de tubos". Associação Brasileira de Metalurgia, Materiais e Mineração. (2016, No Prelo). 